\title{
Effects of generation on memory access
}

\author{
GREG A. PERFETTO, A. ALISON YEARWOOD, JEFFERY J. FRANKS, \\ and JOHN D. BRANSFORD \\ Vanderbilt University, Nashville, Tennessee
}

\begin{abstract}
Prior research has demonstrated that subjects' failure to spontaneously access acquisition information during a problem-solving task results in performance deficits that persist even after the subjects are informed of the relevance of acquisition information (Perfetto, Bransford, \& Franks, 1983). The present experiments examined the hypothesis that these deficits are due to interference from previously generated inadequate solutions to the problems. The results indicated that subjects who generated responses on a first problem-solving trial were able to recall fewer acquisition answers on a second, explicit memory trial than were subjects who read the same Trial 1 responses as experimenter-provided material. This effect seems to be due, at least in part, to greater spontaneous noticing of the relevance of acquisition information by read than by generate subjects.
\end{abstract}

The manner in which past experiences influence processing of current events is an important concern for the psychology of memory. Current theories of memory have attempted to understand this interaction within what might be called a directed-memory paradigm. A crucial feature of the resulting experiments is that subjects are explicitly directed to complete a memory task with reference to some specified set of previous experiences. They are told what they must remember and where the to-beremembered information was last encountered. Note, however, that such explicit memory cues are seldom present outside of a laboratory setting.

Recently, a number of studies have used an alternative spontaneous-access paradigm (e.g., Gick \& Holyoak, 1980; Perfetto, Bransford, \& Franks, 1983; Weisberg, DiCamillo, \& Phillips, 1978). In this paradigm, subjects are presented with problems that can be solved by using recently experienced information. However, they are not explicitly directed to use this information. These studies suggest that spontaneous access of information is not an automatic process, even when the information is both highly relevant and potentially available. Furthermore, these studies indicate that conclusions drawn from experiments conducted under explicit retrieval conditions are not necessarily generalizable to situations that require spontaneous memory access.

In previous work (Perfetto et al., 1983), we tested subjects' spontaneous memory for acquisition information by presenting them with word problems that could be unambiguously solved by using statements presented during an incidental acquisition task. Half of the subjects (in-

This research was supported in part by Contract MDA-903-84-C-0218 from the US Army Research Institute to Jeffery Franks and John Bransford. We would like to thank Jane Kasserman, Larry Jacoby, Fergus Craik, and two anonymous reviewers for comments on earlier versions of this manuscript. Requests for reprints should be sent to Greg Perfetto, Department of Psychology, Vanderbilt University, 134 Wesley Hall, Nashville, TN 37240. formed) were told that the acquisition items would be helpful; the remaining subjects (uninformed) were not. Following the first attempt at problem solving, the subjects were given the same problems again. This time all subjects were told that the acquisition information provided the answers to the problems. On the first problem-solving trial, uninformed subjects were much less likely than informed subjects to access acquisition information and solve the problems. Surprisingly, this deficit carried over to the second, informed trial. It seems that attempts to solve problems while uninformed can lead to deficits in subsequent problem-solving performance, and that these deficits are not eradicated by simply informing subjects about relevant information prior to a second attempt at the problems.

The present study explored a potential reason for this deficit. In the work of Perfetto et al. (1983), during initial problem solving, uninformed subjects generated answers, albeit not very adequate ones, to most of the problems. Perfetto et al. suggested that their subsequent inability to retrieve acquisition information was due to the fact that they were accessing and remembering their own previously generated responses. This could interfere with access to the appropriate acquisition answers. In Experiment 1 we investigated the hypothesis that the deficits on the second, explicit memory test are due to inappropriate accessing of previously generated answers. The rationale for the design rests on the generation effect. Research (e.g., Bobrow \& Bower, 1969; Slamecka \& Graf, 1978) has demonstrated that self-generation enhances memory for information. In the present study, some subjects generated answers to the problems during the first problemsolving trial, while other subjects read the answers produced by the generate subjects. Generated answers should be more likely to be automatically accessed during the subsequent problem solving. Thus, subjects who generated inadequate answers should show greater interference in accessing the acquisition information than subjects who read the answers. 


\section{EXPERIMENT 1}

\section{Method}

Subjects. Subjects were 80 undergraduate students enrolled in an introductory psychology class at Vanderbilt University. They received course credit for their participation.

Materials and Procedure. Materials for this experiment were the 12 problem-clue pairs developed by Perfetto et al. (1983). Subjects were first asked to rate 14 statements for general truthfulness on a 5-point scale (e.g., never true, sometimes true, always true). Twelve of the 14 statements were sentences constructed to provide solutions to the experimental problems. The first and last statements were filler items similar in form to the other statements but unrelated to the experimental problems. Subjects were allowed $20 \mathrm{sec}$ to read and rate each statement. This rating task constituted an incidental acquisition task for problem solutions relevant to the subsequent problem-solving and recall tasks.

Following the acquisition task, a delay of $3 \mathrm{~min}$ was created by collecting the rating sheets and directing subjects to fill out an attendance form. All subjects were then told that they were going to receive some problems. Informed subjects were told that the sentences they had just rated would provide solutions to most of the problems. These instructions were omitted for uninformed subjects. Within the informed and uninformed groups, half of the subjects were assigned to the generate condition, and half to the read condition. Subjects in the generate condition were told to answer the problems and then to rate their answers on a 4-point scale of "goodness" (i.e., poor, inadequate, adequate, good). Each subject in the read condition was provided with the answers to the problems that had been produced by a generate subject. They were told to read the solutions and to rate each on the 4-point goodness scale.

All groups were given problem booklets and answer sheets. The answer sheets were blank for subjects in the generate condition. Read subjects' answer sheets had the corresponding yoked generate subjects' solutions typed on them. The problem booklets were constructed by dividing the 12 experimental problems into two lists of 6 problems equated for difficulty on the basis of prior data. Each booklet contained one set of 6 experimental problems prefaced by 3 filler problems unrelated to the acquisition sentences. Subjects were allowed $40 \mathrm{sec}$ to complete each problem.

During Trial 2 (a recall trial), all subjects received the "informed" instructions, were given new answer sheets and problem booklets, and were told to use the problems as cues for recalling the acquisition sentences. Each subject received the six old problems he/she had seen on Trial 1 as well as six new problems he/she had not previously encountered. The problems were ordered so that no more than two old or new problems appeared in succession, and problems were counterbalanced across the old/new factor. The problem booklets were prefaced with the three filler problems, and subjects were again allowed $40 \mathrm{sec}$ to work on each problem.

\section{Results and Discussion}

Trial 1 solutions produced by generate subjects were scored for congruence with the acquisition answers. Trial 2 data were scored for gist recall of the acquisition sentences. The results are shown in Table 1 .

The data from generate subjects in Experiment 1 were analyzed to determine whether they replicated the find- ings reported by Perfetto et al. (1983), and this was, in fact, the case. A one-way ANOVA confirmed a main effect for the informed/uninformed factor on Trial 1 $[F(1,36)=34.89, p<.001]$, with informed subjects producing more correct answers than uninformed subjects. The Trial 2 analyses confirmed an interaction between the informed/uninformed factor and problem type (old vs. new) $[F(1,36)=20.86, p<.001]$. Informed and uninformed subjects were equivalent in performance on new items. Informed subjects did slightly better on old than on new items, presumably due to their having more time overall to attempt informed retrieval of the answers to the old items. Most importantly, for uninformed subjects, recall was worse for old than for new items. This result replicates the finding that uninformed generate subjects experience interference in the access of the acquisition answers for old problems.

Given this replication, the relative performance of generate versus read subjects on Trial 2 was assessed by a 2 (read/generate) $\times 2$ (informed/uninformed) $\times 2$ (problem type: old/new) ANOVA. Main effects were observed for the informed/uninformed factor $[F(1,72)=$ $23.5, p<.001]$ and problem type $[F(1,72)=5.95$, $p<.05]$. There was also a significant interaction between these two factors $[F(1,72)=21.91, p<.001]$. However, contrary to expectations, the interactions involving the $\mathrm{read} /$ generate manipulation were not significant. Thus, the pattern of results for generate subjects described above also holds for read subjects. Nevertheless, based on our a priori hypothesis, we compared the performance of read and generate subjects for old problems on Trial 2. Generate subjects did recall fewer acquisition answers than did read subjects $[t(38)=1.69, p<.05]$, although this was only marginally significant. Since performance on new items was equivalent across all conditions, this difference on old problems can be attributed to differences between read and generate processing during Trial 1 . Thus, both generate and read subjects showed interference effects on Trial 2 by recalling fewer old than new answers, but the deficit was greater for generate subjects.

As hypothesized, the detrimental effects of previous uninformed problem solving on later, explicit memory tests were more pronounced when subjects generated answers to problems than when the same answers were read as experimenter-provided material. In previous research (e.g., Bobrow \& Bower, 1969; Slamecka \& Graf, 1978; Soraci, 1982), self-generation has been associated with facilitating memory performance, a benefit. The present data suggest that there can also be negative effects, or

Table 1

Mean Proportion of Problems Solved (Trial 1) and Items Recalled (Trial 2) in Experiment 1

\begin{tabular}{|c|c|c|c|c|c|c|c|c|c|c|c|}
\hline \multirow[b]{4}{*}{ Context } & \multicolumn{5}{|c|}{ Read Condition } & \multicolumn{6}{|c|}{ Generate Condition } \\
\hline & \multirow[b]{3}{*}{ Trial 1} & \multicolumn{4}{|c|}{ Trial 2} & & & \multicolumn{4}{|c|}{ Trial 2} \\
\hline & & \multicolumn{2}{|c|}{ Old } & \multicolumn{2}{|c|}{ New } & \multicolumn{2}{|c|}{ Trial 1} & \multicolumn{2}{|c|}{ Old } & \multicolumn{2}{|c|}{ New } \\
\hline & & Mean & $S E$ & Mean & $S E$ & Mean & $S E$ & Mean & $S E$ & Mean & $S E$ \\
\hline Informed & & .79 & .041 & .73 & .038 & .67 & .056 & .82 & .041 & .73 & .04 \\
\hline Uninformed & & .56 & .065 & .70 & .042 & .24 & .04 & .41 & .055 & .72 & .058 \\
\hline
\end{tabular}


costs, associated with self-generation. Generating responses may accentuate interference with subjects' ability to flexibly access previously experienced information.

These results are consistent with the hypothesis that enhanced memorability of self-generated answers results in increased interference with the access of acquisition information. However, the experiment provided no direct evidence for the superior memorability of the selfgenerated answers. Experiment 2 was designed to provide this evidence by asking subjects to recall on Trial 2 both the acquisition answers and the answers that were either generated or read during Trial 1. It was expected that generate subjects would have better memory for the latter answers and worse recall for the acquisition answers than would read subjects.

\section{EXPERIMENT 2}

\section{Method}

Subjects. Subjects were $\mathbf{4 0}$ undergraduate volunteers enrolled in an introductory psychology class at Vanderbilt University. They received course credit for their participation.

Materials and Procedure. The materials and procedure were the same as those of Experiment 1, with the following exceptions. All subjects were uninformed during Trial 1 problem solving. Furthermore, all 12 experimental problems were presented on both Trial 1 and Trial 2 (i.e., all problems were old). On Trial 2, all subjects were instructed to recall both the relevant acquisition sentence and the Trial 1 response for each problem, in any order. Finally, in this experiment a questionnaire was used to assess whether subjects spontaneously noticed the relevance of the acquisition answers during Trial 1.

\section{Results and Discussion}

During Trial 1 , generate subjects solved $23.7 \%$ of the problems. This level of performance is comparable to that of uninformed generate subjects in Experiment 1. The pattern of recall for acquisition answers on Trial 2 also essentially replicates the findings from Experiment 1 . In particular, read subjects recalled significantly more acquisition items than did generate subjects, with mean proportions of $.56(S E=.036)$ and $.44(S E=.045)$, respectively $[t(28)=2.1, p<.05]$.

The data of primary interest involved subjects' recall of Trial 1 answers, which they either read or generated. It was expected that generated answers would be better recalled. Unfortunately, both generate and read subjects had near-perfect memory for the Trial 1 answers, with mean recall rates of $97.5 \%$ and $98.7 \%$, respectively. This ceiling effect precludes any strong conclusions concerning the relationship between greater memorability of generated answers and Trial 2 recall deficits.

However, the data do provide evidence for an alternative reason for read subjects' recalling more old items than did generate subjects, and for the general hypothesis that retrieval of inappropriate Trial 1 answers interferes with recall of acquisition answers during Trial 2. On the postexperimental questionnaire, read subjects reported noticing the relevance of acquisition answers for the problems on Trial 1 more often than did generate sub- jects. Subjects who spontaneously noticed the relevance at some point during Trial 1 could have retrieved the acquisition answers for the remaining Trial 1 problems and thus attenuated subsequent interference effects on these items. If read subjects were more likely to spontaneously notice the relevance of acquisition during Trial 1, then this could account, at least in part, for their superior performance on old items during Trial 2. This interpretation is supported by recall data.

Spontaneous noticing of the relevance of acquisition answers can occur at any time during Trial 1 . It is likely that over the course of Trial 1 , increasing numbers of subjects will have attained spontaneous access. Once access has been achieved, the remaining Trial 1 problems will be associated with the appropriate acquisition answers, and these answers will be readily available for Trial 2 recall. If our read subjects did indeed have an advantage in attaining spontaneous access, their observed superiority in recall of acquisition answers for old items should be especially apparent for items that occurred late in the list during Trial 1 . This hypothesis was examined by comparing Trial 2 recall of acquisition answers related to the first six problems encountered during Trial 1 with recall of answers for the final six problems. Since subjects were administered different random orders of problems on Trials 1 and 2, the serial position of a problem on Trial 1 was unrelated to the position of that problem on Trial 2. For generate subjects, recall was equivalent for problems occurring in the first and second halves of the Trial 1 order, with mean proportions of $.44(S E=.05)$ and .45 $(S E=.063)$, respectively. Read subjects, on the other hand, recalled fewer answers for first-half problems (.50, $S E=.05)$ than for second-half problems $(.63, S E=.05)$. The recall advantage of read subjects over generate subjects was not significant for first-half problems $[t(38)=$ $.86, p>.05]$, but was significant for second-half problems $[t(38)=2.24, p<.05]$.

This result supports the hypothesis that the recall advantage demonstrated by read subjects was due, at least in part, to enhanced spontaneous access of acquisition answers on Trial 1. This finding is also consistent with the general hypothesis that inadequate Trial 1 answers interfere with access to acquisition answers during Trial 2 . If the latter answers are accessed for problems during Trial 1, the interfering effects of any inadequate answers either generated or read for those problems should be attenuated. It may also be the case that greater memorability of generated than of read answers also contributes to the recall disadvantage of the generate subjects, but direct support for this hypothesis awaits further work.

Finally, why were read subjects more likely than generate subjects to spontaneously notice the relevance of the acquisition answers during Trial 1? Bandura (1977) suggested that observational processes (e.g., reading and evaluating solutions) make fewer demands on a person's attention faculties than does active involvement, with the result being a broader perspective on the situation in the 
former case. In the present experiments, generate subjects were required to actively devise, as well as evaluate, solutions. Read subjects, in contrast, were given the less demanding task of simply evaluating another person's solution. Thus, read subjects may have had a broader perspective on the situation. As a result, they may have been more sensitive to potentially available relationships such as that between the acquisition answers and the problems. Direct support for this attentional-demand interpretation will require further experiments.

\section{REFERENCES}

Bandura, A. (1977). Social learning theory. Englewood Cliffs, NJ: Prentice-Hall.
Bobrow, S. A., \& Bower, G. H. (1969). Comprehension and recall of sentences. Journal of Experimental Psychology, 80, 455-461.

Gick, M. L., \& HolyoaK, K. J. (1980). Analogical problem solving. Cognitive Psychology, 12, 306-355.

Perfetto, G. A., Bransford, J. D., \& Franks, J. J. (1983). Constraints on access in a problem solving context. Memory \& Cognition, 11, 24-31.

Slamecka, N. J., \& Graf, P. (1978). The generation effect: Delineation of a phenomenon. Journal of Experimental Psychology: Human Learning \& Memory, 4, 592-604.

SORACI, S. A. (1982). An exploration of generate processing. Unpublished doctoral dissertation, Vanderbilt University, Nashville, TN.

Weisberg, R., DiCamillo, M., \& Phillips, D. (1978). Transferring old associations to new situations: A nonautomatic process. Journal of Verbal Learning \& Verbal Behavior, 17, 219-228.

(Manuscript received for publication November 28, 1986.) 